\title{
IMPROVING ULTRA WIDEBAND (UWB) SYSTEM BY MODIFIED RANDOM COMBINATION OF PULSES
}

\author{
Vikas Goyal* - B.S Dhaliwal \\ Department of Electronics and Communication Engineering, Guru Kashi University, Talwandi Sabo, India
}

\begin{tabular}{l} 
ARTICLE INFO \\
\hline Article history: \\
Received: 15.06 .2016$. \\
Received in revised form: 01.01 .2017$. \\
Accepted: 14.01 .2017$. \\
\hline Keywords: \\
UWB \\
PPM \\
PPM-TH \\
Multipath channel \\
Receiver \\
Gaussian
\end{tabular}

\section{Introduction}

The type of pulse shape to be used for the UWB systems is very important for achieving high data rates.

It uses Gaussian pulse shape and its derivative for the signal generation [1]. The UWB systems with central frequency $\left(f_{\mathrm{C}}\right)$ greater than $2.5 \mathrm{GHz}$ require a bandwidth of at least five hundred $\mathrm{MHz}$, while the UWB systems with the central frequency less than

\begin{abstract}
:
Ultra-Wideband (UWB) technology uses very narrow pulses of nano-seconds duration to provide very high data rates. The pulses to be used for the UWB are very important and should meet the emission mask regulatory requirements to get good performance when transmitted over the channel. To meet these requirements, a combination of higher order derivatives of Gaussian pulses can be considered to form a combined pulse instead of transmitting them separately. The main aim of this paper is to improve the performance of Ultra Wideband (UWB) multiple access modulation system with a newly Proposed combined pulse, generated using a modified random pulse combining of the first five Gaussian derivative pulses with optimal shaping factors as basic functions. In order to meet the UWB power spectrum regulations, the power spectral utilization of the newly designed pulse is calculated and the bit error rate performance of the pulse position modulation time hopping system using the newly proposed pulse is analyzed in a multi-path channel environment using RAKE receiver. The simulation results carried out in MATLAB software shows that the new proposed combined pulse has higher pulse energy matching the spectral limits of $U W B$ and an improved bit error rate performance as compared to the previous literature results.
\end{abstract}

2.5 $\mathrm{GHz}$ should have the fractional bandwidth (Bandwidth/ central frequency) of at least 0.2 [2].

For each frequency band the Federal Communications Commission (FCC) has assigned the allowed effective isotropic radiated power (EIRP) [3] for UWB devices and should be below -41.3 $\mathrm{dBm} / \mathrm{MHz}$ in the frequency range of 3.1 to $10.6 \mathrm{GHz}$. In the past researches, several pulse shapes found in the literature for UWB communication but the

\footnotetext{
* Corresponding author. Tel.: +91 09872153694

E-mail address: vikas312002@gmail.com
} 
optimal choice of a pulse shape depends on the pulse time and frequency response and on the application. Research paper [4] explained that Impulse radio can be used for ultra wideband transmission considering a Gaussian monocycle pulse as an option for the generation of the ultra-wideband signal. Another research paper [5] studied the pulse shapes that can be used for the UWB communication systems. Ultrawideband pulse design method has been proposed in [6] that made use of a linear pulse combination waveform of the sub-band signals.

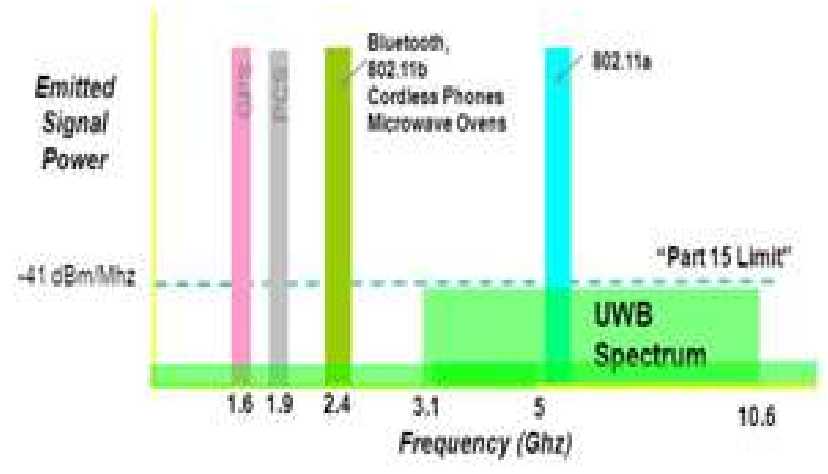

Figure 1. UWB Spectrum

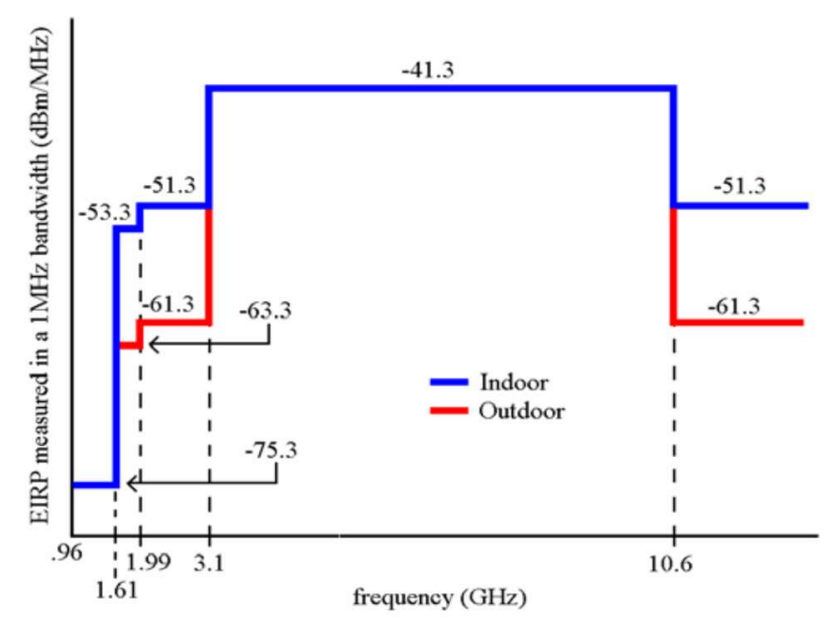

Figure 2. EIRP/emission mask limits of UWB

A linearly combining two monocycles using different pulse shapes is described in [7] to satisfy the radiated power limits of ultra wideband transmission. A combination of Gaussian derivatives over definite sub bands to increase the power spectrum utilization is described by taking the pulse shaping factors and the numbers of derivatives by the trial and error method to match the emission mask [8].

The UWB pulses and their power spectral efficiency is generated by the combining the multiple monocycle pulses of first, second, third and fifth Gaussian derivatives with their inverse polarities to meet the emission mask criteria in [9]. A pulse shaping techniques is investigated the UWB waveforms by using three different pulse shapes i.e. Gaussian monocycles, fifth derivative and by combining the Gaussian monocycle and its higher order derivatives and measured their power spectral densities and efficiency to satisfy the emission mask [10]. A linear combining method of waveforms for matching the emission mask criteria of the UWB systems was also presented in [11].

A pulse design technique based on the linear combination of Gaussian doublet pulses is presented in [12] and it was found that the proposed pulse showed better spectral power efficiency as compared to the conventional pulse shapes. A new UWB pulse shaping method has been presented in [13] [14] that divides the entire spectrum into regions and in each region the Gaussian pulse and its derivatives are combined using least square error combination techniques having same shaping factors in order to improve the spectral mask fitting.

Research paper [15] illustrates direct sequence impulse UWB radio modulation systems. Article [16] presented the applications of the ultra-wideband communication systems. IEEE 802.15.3a UWB multipath Channel Model described in [17] and [18] for Ultra wideband Indoor Communication. Article [19] [20] and [21] presented the RAKE receiver with Maximal Ratio Combining for ultra wideband communication systems. The performance of UWB indoor channel model for all channels with different modulation scheme is also investigated in [22].

In this research article, we will propose a new combined pulse system method using random pulse combining technique using the first five Gaussian derivative pulses with optimal shaping factors as basic functions. Then we will analyze the bit error rate performance of the new proposed pulse position modulation time hopping system in a multi-path channel environment. We will use RAKE receiver maximal ratio combining technique. The simulation results are carried out in MATLAB software.

\section{Proposed approach and work done}

As the emission masks requirements set are very important to be met by the regulation authorities by the pulse to be considered for the ultra-wideband transmission. To find a suitable pulse for the ultrawideband system will give optimal results when transmitted over the channel and that are in 
accordance with the EIRP power limits of $41.3 \mathrm{~dB} / \mathrm{MHz}$ in $3.1 \mathrm{GHz}$ to $10.6 \mathrm{GHz}$ frequency range. A new pulse combined method can be considered where a pulse consists of combined Gaussian derivatives and it can result in an effective performance over the multipath channel.

The basic Gaussian pulse [3] is given as

$$
P(t)=\frac{A}{\sqrt{2 \pi \sigma^{2}}} e^{-\frac{t^{2}}{2 \sigma^{2}}} \quad-\infty<t<\infty
$$

Where A is the amplitude, $\sigma$ is called as the standard deviation of the pulse and the optimal pulse shaping factor $(\alpha=2 \sigma \sqrt{ } \pi)$.

By differentiating with time ( $t$ ), we can get the First Gaussian derivative as:

$$
P(t)=\frac{A}{\sqrt{2 \pi \sigma^{2}}}\left(1-\frac{t^{2}}{2 \sigma^{2}}\right) e^{-\frac{t^{2}}{2 \sigma^{2}}} \quad-\infty<t<
$$

The Fifth Gaussian derivative as:

$$
\begin{aligned}
& P(t)= \\
& A\left(-\frac{t^{5}}{\sqrt{2 \pi} \sigma^{11}}+\frac{10 t^{3}}{\sqrt{2 \pi} \sigma^{9}}-\frac{15 t^{3}}{\sqrt{2 \pi} \sigma^{7}}\right) e^{-\frac{t^{2}}{2 \sigma^{2}}} \\
& -\infty<t<\infty
\end{aligned}
$$

Rewriting the equation (1) we get:

$$
P(t)={ }_{-}^{+} \frac{A \sqrt{2}}{\alpha} e^{-\frac{2 \pi t^{2}}{\alpha^{2}}}
$$

Where $\alpha^{2}=4 \pi \sigma^{2}, \alpha=$ Pusle shape factor

Also, the higher derivative of the Gaussian pulse equation can be expressed as:

$$
P^{(n)}(t)=-\frac{n-1}{\sigma^{2}} P^{(n-2)}(t)-\frac{t}{\sigma^{2}} P^{(n-1)}(t)
$$

Fourier transform (FT) of the $n^{\text {th }}$ Gaussian pulse derivative can be given as:

$$
\left|P^{(n)}(f)\right|=A(j 2 \pi f)^{n} e^{\left(-\frac{(2 \pi f \sigma)^{2}}{2}\right)}
$$

The amplitude spectrum of the $\mathrm{n}^{\text {th }}$ order Gaussian derivative is given as:

$$
\left|P^{(n)}(f)\right|=A(j \pi f)^{n} e^{\left(-\frac{(2 \pi f \sigma)^{2}}{2}\right)}
$$

The frequency $f_{\mathrm{m}}$ at which the highest value of the above equation achieved is the peak emission frequency $f_{M}$, and it may be obtained by differentiating the above equation with respect to frequency and setting it equal to zero.

$$
\begin{aligned}
& \frac{d\left|P^{(n)}(f)\right|}{d f}= \\
& A(j \pi f)^{n-1} 2 \pi e^{\left(-\frac{(2 \pi f \sigma)^{2}}{2}\right)}\left(n-(2 \pi f \sigma)^{2}\right)
\end{aligned}
$$

The peak emission frequency should ensure that

$$
2 \pi f_{M} \sigma=\sqrt{n}
$$

Therefore, the maximum value of the amplitude spectrum is given as:

$$
\left|P_{n}\left(f_{M}\right)\right|=A\left(\frac{\sqrt{n}}{\sigma}\right)^{n} e\left(-\frac{n}{2}\right)
$$

The normalized Power Spectral Density of the pulse shape is given as:

$$
\begin{aligned}
\left|P S D_{n}(f)\right| & =\frac{\left|P_{n}(f)\right|^{2}}{\left|P_{n}\left(f_{M}\right)\right|^{2}} \\
& =\frac{(2 \pi f \sigma)^{2 n}}{n^{n}} \frac{e^{-(2 \pi f \sigma)^{2}}}{e^{-n}}
\end{aligned}
$$


The transmitted signal's Power Spectral Density $\left(P S D_{t}\right)$ is given by:

$$
\begin{aligned}
\left|P S D_{t}(f)\right| & =A_{\max }\left|P S D_{n}(f)\right| \\
& =A_{\max } \frac{(2 \pi f \sigma)^{2 n}}{n^{n}} \frac{e^{-(2 \pi f \sigma)^{2}}}{e^{-n}}
\end{aligned}
$$

Where $A_{\max }$ is the peak allowed $P S D$ emission mask. By solving the above equation, we can find the optimal values of the Gaussian pulse derivatives that satisfy the UWB emission mask limits.

To get more improved result, we will combine the higher order derivatives of the Gaussian pulse to generate a single waveform that follows the EIRP radiation limits described above very closely.

The combined pulse can be given as:

$$
P(t)=\sum_{n=1}^{N} C_{n} P^{n}\left(\alpha_{n}, t\right)
$$

Where $n$ denotes the derivative order of the Gaussian pulse, $\alpha$ denotes the Pulse shape factor and $C_{n}$ denotes the set of optimal coefficients.

To find the optimal set of coefficients we will use the proposed method (Modified random combination) to combine the higher order Gaussian derivatives explained below:

Step 1. Generate the set of different Gaussian derivatives $(i=1: n)$.

Step 2. Apply the pulse shape factors to the generated Gaussian derivatives.

Step 3. Generate the UWB Emission Mask with defined EIRP limits.

Step 4. Set the number of attempts to find the most optimal coefficient set closely meeting UWB EIRP/emission limits.
Step 5. Initialization - Initialize the random number generator, coefficients set and number of attempts (m)

Step 6. Random Generation - Generate the random coefficients set for the Gaussian derivatives.

Step 7. Generate the combined pulse using Gaussian derivatives and the generated random set in step 6 .

Step 8. Find the Power Spectral Density (PSD) of combined Pulse.

Step 9. Check whether the obtained PSD of the combined pulse meet the UWB emission mask limits.

Step 10. If the emission mask/EIRP limits are not satisfied then repeat the step no.6 and obtain another set of random coefficients or otherwise go to step 11 .

Step 11. If the limits are satisfied, record the set of coefficients and its related Power.

Step 12. If it is the first obtained set meeting the limits then go to step no.14, otherwise go to step no. 13 .

Step 13. Compare the Power of present set with the previous present/recorded set to see which set has the better matching of the emission mask limits.

Step 14. Record the set as having the highest power, then go to step no. 15.

Step 15. Mark the set of coefficients as optimal set C with highest power.

Step 16. Go to step no.5 for another attempt and repeat the process to obtain the set of coefficients satisfying the limits more closely.

Step 17. After running all attempts, we will get the most optimal set of coefficients $C$ having the highest power, which meets the emission mask limits very closely.

Step 18. Obtain the combined pulse using the most optimal set C. 


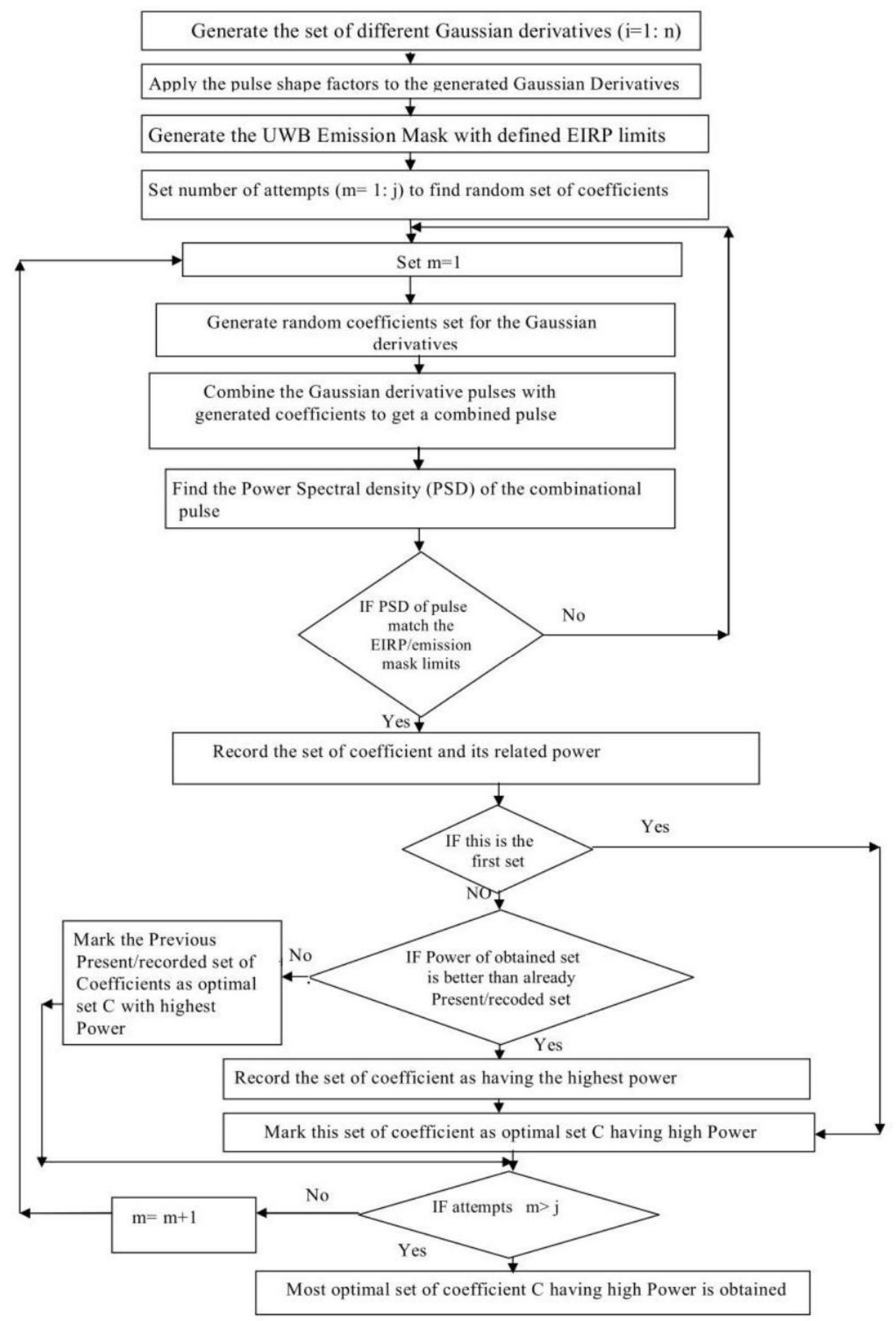

Figure 3. Proposed Approach for Pulse Combining 


\section{The UWB system using proposed method}

For reducing the interference from the other transmission devices and providing the multiple access capability a technique called time-hopping
(TH) is used with the pulse position modulation technique in which the pulses can be sent earlier or delayed in time for modulating the signal [23].

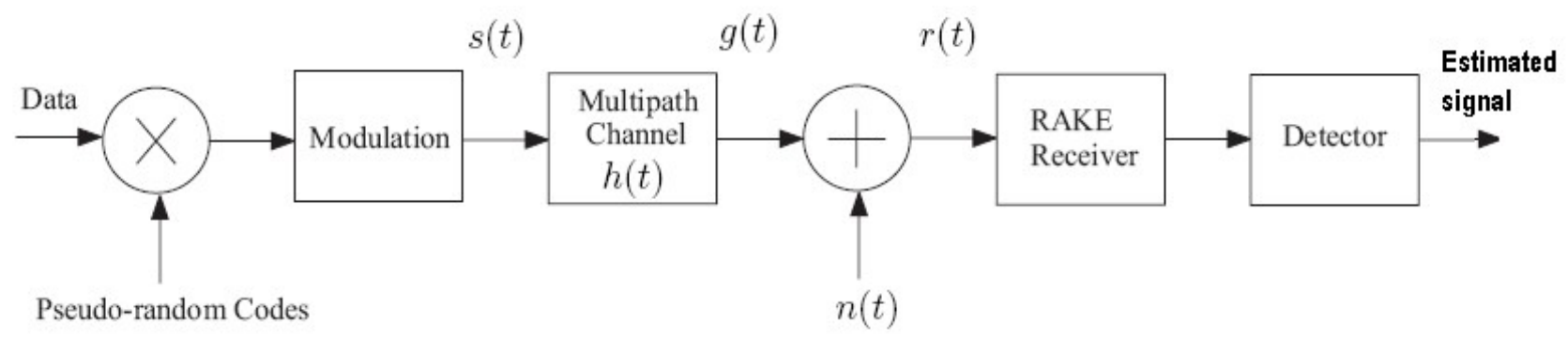

Figure 4. The UWB multiple access system using combined pulse

In this research article, we have considered the UWB transceiver shown in the Fig. 4 using a randomizing technique called time hopping (TH) pulse position modulation (PPM) technique and the proposed optimal combined pulse shapes.

The bits having information are processed using pulse position modulation technique.

Where $r(\mathrm{t})$ is the received signal and $m(\mathrm{t})$ is correlation mask.

The binary bits to be transmitted are added with a user specific pseudo code before sending them to the pulse position modulator. These pulses sequence are positioned at $j T_{s}$ in a specific time hopping slot according to the code $\left(c_{j} T_{c}\right)$ along with the pulse shift introduced by the pulse position modulator to modulate the bits [24]. The signal at the output of the transmitter is represented as

$$
s(t)=\Sigma_{j=-\infty}^{+\infty} p\left(t-j T_{s}-c_{j} T_{c}-a_{j} \varepsilon\right)
$$

Where, $c_{j} T_{c}=$ shift in time which is introduced by the Time hopping code, $a_{j} \varepsilon=$ delay shift which is added by the PPM modulator and $T_{s}$ is frame time.

$$
g(t)=S(t) \cdot h(t)
$$

Where, $g(t)$ is the useful signal at the receiver and $n(t)$ is an additive noise assumed to be the Gaussian process.

The output of the transmitter is sent over the ultrawideband multipath channel (IEEE 802.15.3a) [26] [27]. This impulse response of the channel can be of four types (CM1, CM2, and CM3) with defined user parameters given in the Table 1 .

\begin{tabular}{|c|c|c|c|}
\hline $\begin{array}{l}\text { Channel } \\
\text { Characteristics }\end{array}$ & $\begin{array}{l}\text { Case A } \\
\text { LOS } \\
(0-4 \mathrm{~m})\end{array}$ & $\begin{array}{l}\text { Case B } \\
\text { NLOS } \\
(0-4 \mathrm{~m})\end{array}$ & $\begin{array}{l}\text { Case C } \\
\text { NLOS } \\
(4-10 \mathrm{~m})\end{array}$ \\
\hline $\begin{array}{l}\text { Cluster arrival rate } \\
\Lambda(1 / \mathrm{ns})\end{array}$ & 0.0233 & 0.4 & 0.0667 \\
\hline $\begin{array}{l}\text { Ray arrival rate } \\
\lambda(1 / \mathrm{ns})\end{array}$ & 2.5 & 0.5 & 2.1 \\
\hline $\begin{array}{l}\text { Cluster delay } \\
\text { factor } \Gamma(1 / \mathrm{ns})\end{array}$ & 7.1 & 5.5 & 14.0 \\
\hline $\begin{array}{l}\text { Ray delay factor } \\
\gamma(1 / \mathrm{ns})\end{array}$ & 4.3 & 6.7 & 7.9 \\
\hline $\begin{array}{l}\text { Standard deviation } \\
\text { of cluster } \\
\text { lognormal fading } \\
\sigma_{1}(\mathrm{~dB})\end{array}$ & 3.3941 & 3.3941 & 3.3941 \\
\hline $\begin{array}{l}\text { Standard deviation } \\
\text { of ray lognormal } \\
\text { fading } \sigma_{2}(\mathrm{~dB})\end{array}$ & 3.3941 & 3.3941 & 3.3941 \\
\hline $\begin{array}{l}\text { Standard deviation } \\
\text { of lognormal } \\
\text { shadowing term } \sigma_{j} \\
\text { (dB) }\end{array}$ & 3 & 3 & 3 \\
\hline
\end{tabular}

Table 1. UWB IEEE802.15.3a channel parameters

Where, CM1 used for a line of sight less than $4 \mathrm{~m}$, CM2 for non-line of sight less than 4m and CM3 for non-line of sight, 4-10 $\mathrm{m}$ [28].

In the Multipath channel (IEEE 802.15.3a) model all the components of the multipath and the path ray within each of the group reaches the receiver in clusters or groups along with the Poisson distribution. 
The impulse response of the channel can be represented as:

$$
h(t)=\Sigma_{l-1}^{F} \Sigma_{n-1}^{P} \alpha_{n l} \delta\left(t-T_{l}-\tau_{n l}\right)
$$

where a number of clusters are represented by $F$ and the paths within the group are represented by P. $\alpha_{n l}$ is the $\mathrm{n}^{\text {th }}$ multipath gain. $T_{l}$ and $\tau_{n l}$ gives the delay. Transmitted signal through the channel is received by the rake receiver. The rake receiver shown in Fig. 5 consists of a matched filter that is matched to the transmitted waveform and a tapped delay line that matches with the impulse response of the channel. It uses correlators and delays to spread out the individually delayed multipath signals. Every signal is delayed as per the peaks founded in the received signal. Then each component is separately coded and combined at the end using a maximum ratio combining technique in which the outputs of the correlator are weighted and each branch signal is multiplied with weighting factor (proportional to the signal amplitude) so that the outputs responding to strong signals are given more weight than the weak signals to have higher signal to noise ratio (SNR) in a multipath environment. Based on this the estimated combined signals are found out by the detector.

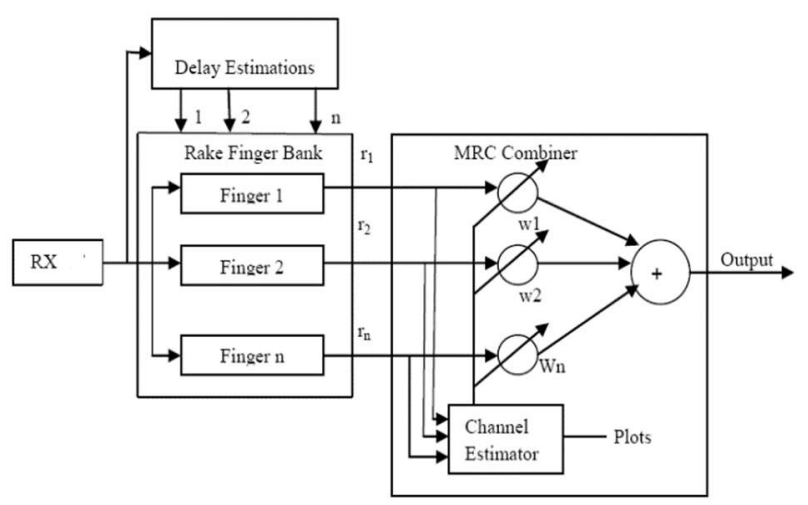

Figure 5. RAKE Receiver

The correlation mask can be expressed as:

Mask

$=p_{0}\left(t-\tau-c_{j} T_{c}\right)$ and $p_{0}\left(t-\tau-c_{j} T_{c}-a j \varepsilon\right)$

where,

$=p_{0}\left(t-\tau-c_{j} T_{c}\right)$ and $p_{0}\left(t-\tau-c_{j} T_{c}-a j \varepsilon\right)$

are the correlation signals used to match the transmission signal for binary bit 0 and bit 1 with pulse shift respectively.
Fig. 6. describes the Receiver structure used for the system model using pulse position modulation and time hopping multiple access technique where the received signal delayed multipath components are correlated with the transmitted signal.

The receiver configurations [19] considered are the Ideal or All RAKE which considers all the multipath delay components, Selective RAKE selects the best paths from all the multipath signals and Partial RAKE takes the first arrived multipath into consideration.

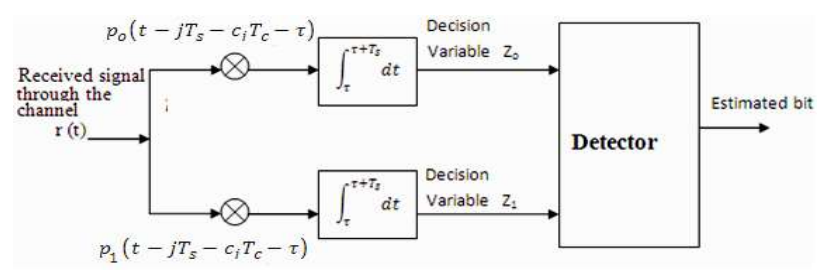

Figure 6. Receiver structure for the system

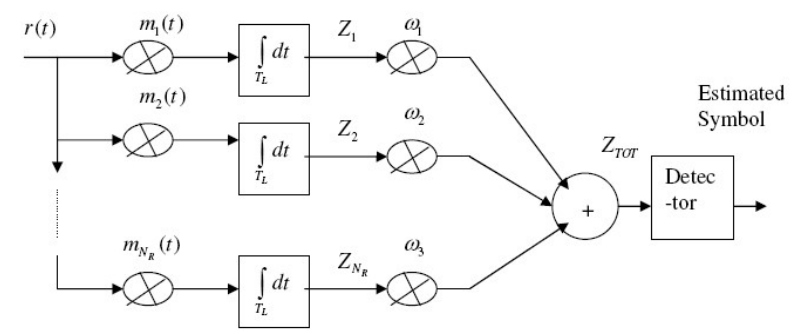

Figure 7. RAKE Receiver with parallel correlators

The received signal can be represented as:

$$
r(t)=g(t)(t)+n(t)
$$

where $g(t)$ is the useful signal at the receiver and $\mathrm{n}(\mathrm{t})$ is additive gaussian noise (AWGN) [25] assumed to be gaussian.

$$
g(t)=\alpha S_{m}(t-\tau)
$$

where, $\alpha$ is the channel gain dependent on the distance between transmitter and receiver. The above rake receiver can be represented in a simple manner using a single correlator given below:

$$
\begin{aligned}
Z_{\text {Tot }} & =\int_{\tau}^{T_{L}} r(t) m_{r}(t) \mathrm{dt} \\
& =\int_{\tau}^{T_{L}} r(t) m\left(t-\tau_{j}\right) \omega_{L}
\end{aligned}
$$


$\omega_{\mathrm{L}}$ denotes the weight factors corresponding to the aplitude of the transmitted signals.

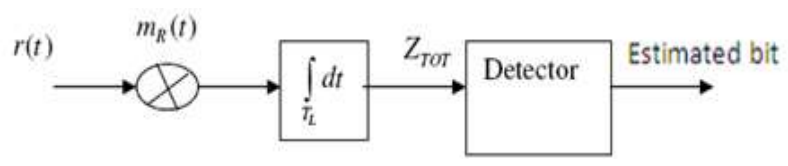

Figure 8. Rake Receiver structure

\section{Experimental simulations}

The above described method of generating an optimal pulse described in the proposed method is created and simulated using Matlab software. Using the bisection method, we can find the optimal values of pulse shape values by finding the roots of equation (12) for higher order derivatives of the Gaussian pulse.

Table 2. Optimal Pulse shape factors values

\begin{tabular}{|l|c|c|}
\hline Derivative & $\begin{array}{c}\text { Pulse shape } \\
\text { value }(\alpha) \text { in } \\
\text { seconds (s) }\end{array}$ & $\begin{array}{c}\text { Peak } \\
\text { Frequency } \\
\text { (GHz.) }\end{array}$ \\
\hline First & $0.1162 \times 10^{-9}$ & 4.885 \\
\hline Second & $0.1363 \times 10^{-9}$ & 5.886 \\
\hline Third & $0.1522 \times 10^{-9}$ & 6.387 \\
\hline Fourth & $0.1657 \times 10^{-9}$ & 6.887 \\
\hline Fifth & $0.177 \times 10^{-9}$ & 7.139 \\
\hline
\end{tabular}

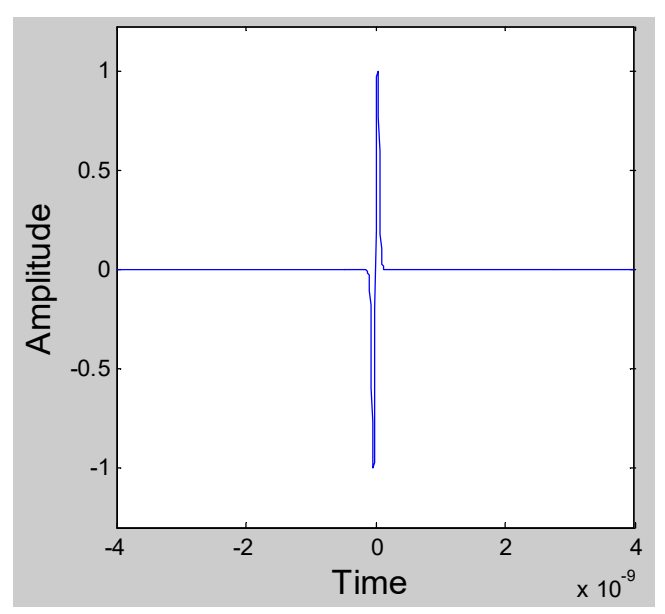

Figure 9. Pulse Shape of the 1st Gaussian derivative Pulse using optimal pulse factor

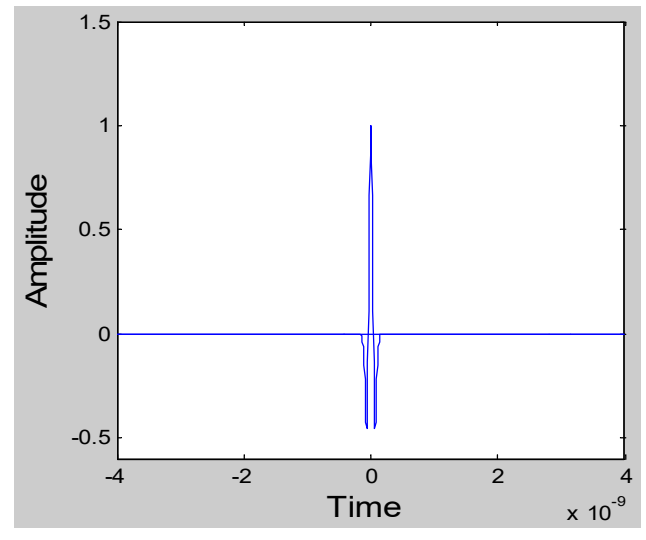

Figure 10. Pulse Shape of the 2nd Gaussian derivative Pulse using optimal pulse factor

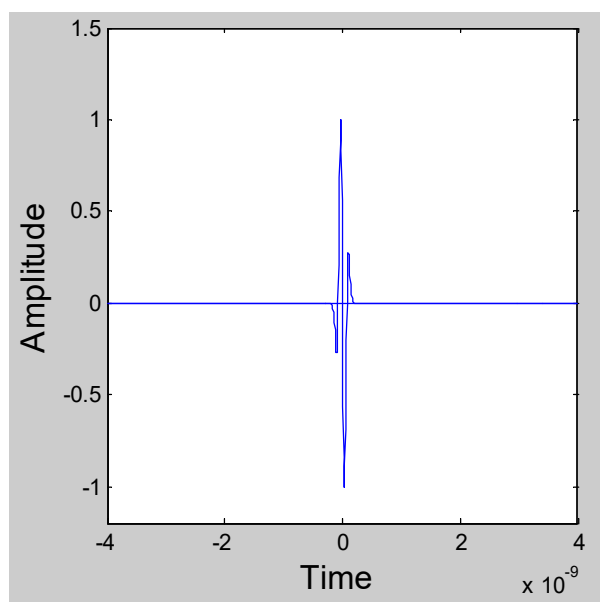

Figure 11. Pulse Shape of the 3rd Gaussian derivative Pulse using optimal pulse factor

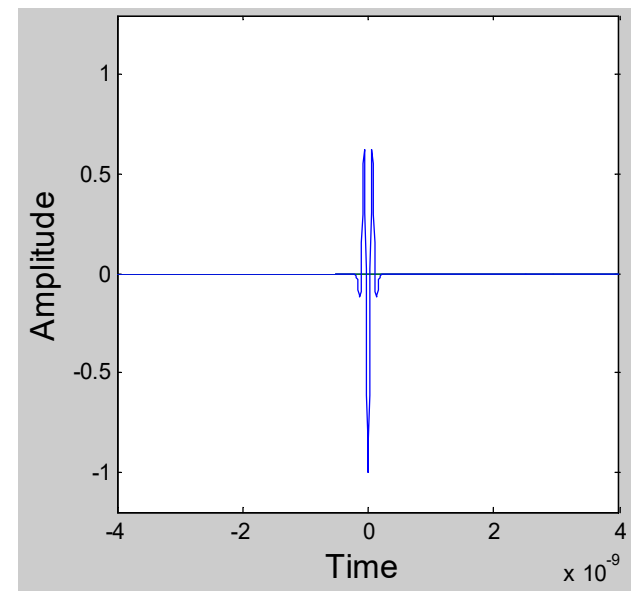

Figure 12. Pulse Shape of the 4th Gaussian derivative Pulse using optimal pulse factor 


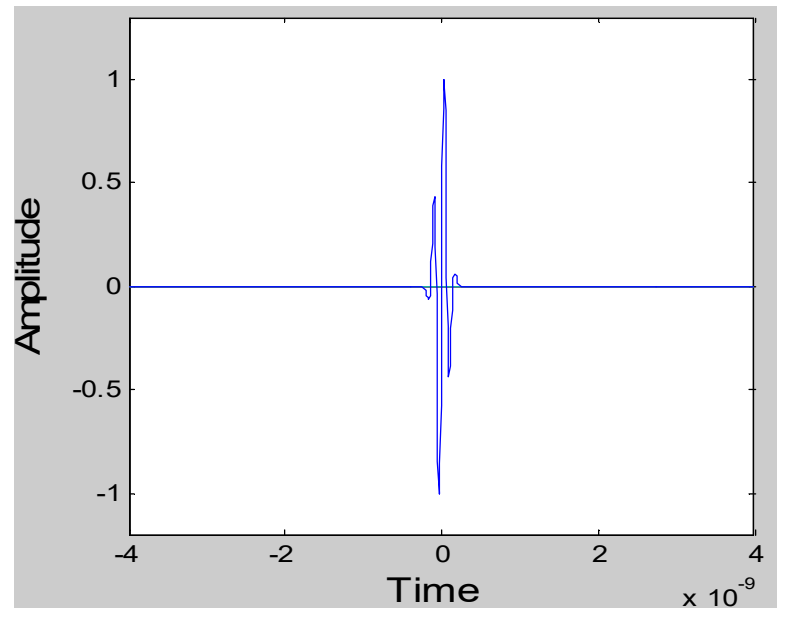

Figure 13. Pulse Shape of the 5th Gaussian derivative Pulse using optimal pulse factor

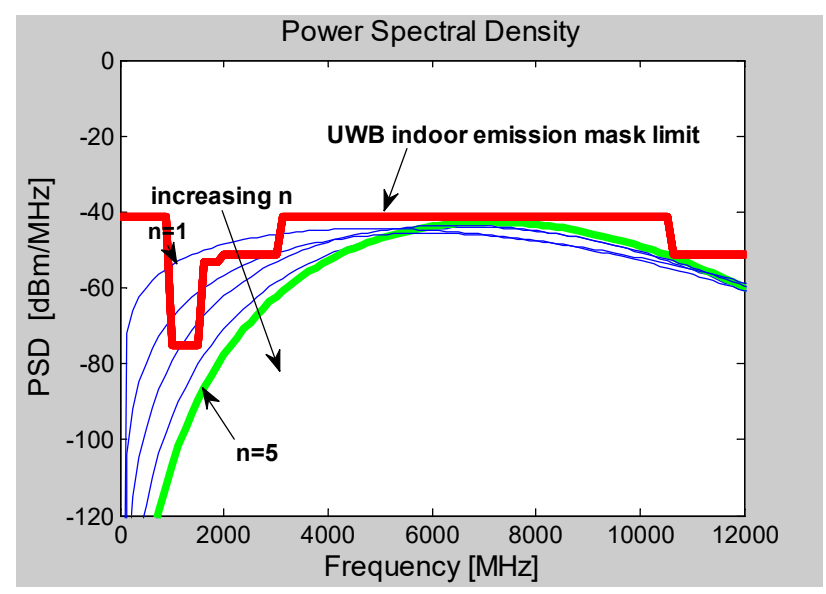

Figure 14. Power Spectral density of the Gaussian derivatives for obtained pulse shape factor value

It is found that the PSD of the $5^{\text {th }}$ order Gaussian derivative shown by green curve closely fits the UWB emission mask as compared to the other derivatives. Therefore, we take the $5^{\text {th }}$ order derivative of the Gaussian pulse as a standard pulse and consider up to $5^{\text {th }}$ derivatives for making an optimal combined pulse in our experimental work. The proposed combined pulse is generated by combining the first five Gaussian derivative pulses with the obtained optimal pulse shape factors as shown in Fig. 15. and its power spectral density (PSD) closely meeting the emission mask requirements is shown in Fig. 16.

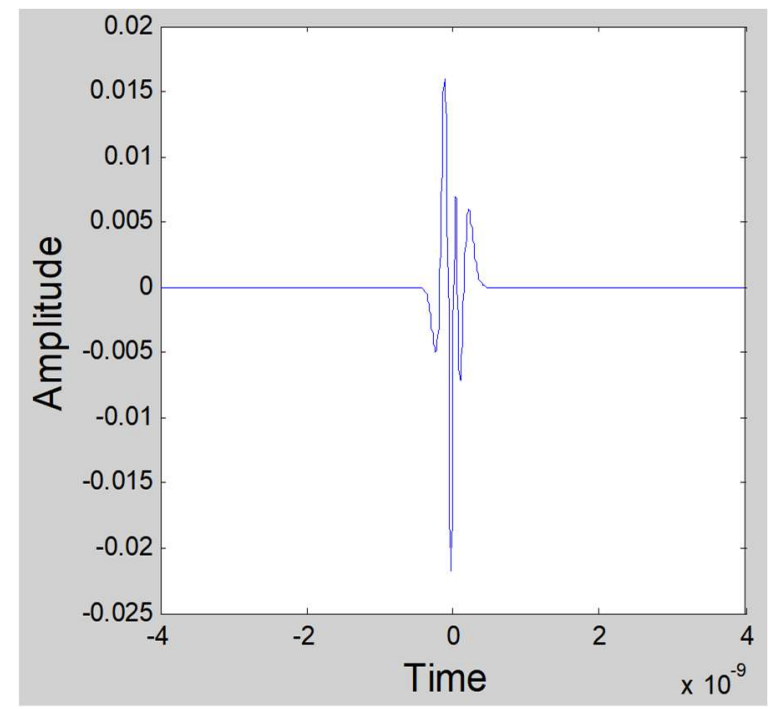

Figure 15. Proposed Combined Pulse

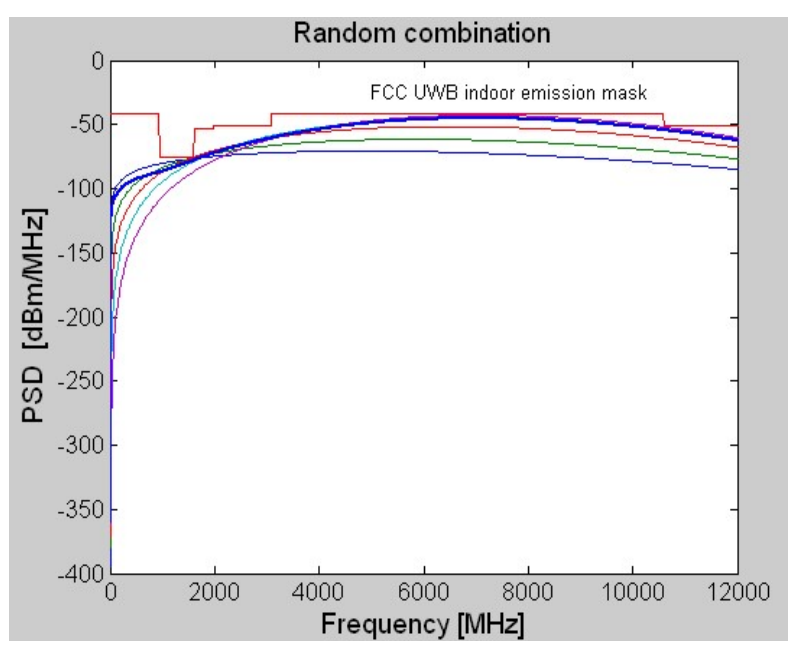

Figure 16. PSD of Proposed Combined Pulse

The above figure shows that the power spectral density (PSD) of the optimal pulse is shown by the dark blue curve following the emission limit requirements effectively as in comparison to the individual PSD of the first five derivatives using optimal values given in the table 2 with the Power Spectral Utilization (Percentile ratio of the spectral power of the pulse transmitted and the spectral power of the emission mask in $3.1 \mathrm{GHz}$ to $10.6 \mathrm{GHz}$ frequency range) of nearly $85 \%$.

The Power Spectral Utilization results obtained using the proposed combined pulse are better than as compared to the results presented in [8] with the shaping factor selected by trial and error method as $1.5 \times 10^{-9} \mathrm{~s}$ for the first derivative and $0.35 \times 10^{-9} \mathrm{~s}$ from the second to the fifteenth derivatives. The results obtained by our method using optimal pulse 
values also show significant improvement as compared to the proposed pulse and the fifth-order derivative with spectral efficiency of $57.40 \%$ by linear combination of the Gaussian doublet pulses in [12], $60-70 \%$ by the combination of the Gaussian pulse and its derivatives in [10] and the power spectral utilization of $35 \%$ and $48.52 \%$ stated for the pulse in [30].

The obtained optimal pulse shows better results as compared to results presented in paper [7] by linearly combining two first-order derivatives of the Gaussian pulses using different pulse shaping values of 0.049 x $10^{-9} \mathrm{~s}$ and $0.048 \times 10^{-9} \mathrm{~s}$. Also, the emission matching attained by our proposed method shows an improvement using the optimal pulse factor values for the first five Gaussian derivatives as compared to the same pulse factor value of $0.7 \times 10^{-9} \mathrm{~s}$ for the derivatives given in [9].

The Ultra wideband transceiver system model is also simulated using the combined proposed pulse and standard Gaussian pulse fifth derivate pulse using the optimal pulse shape factors for Time hopping pulse position multiple access modulation system in the multipath channel environment to calculate the bit error rate (BER) using the parameters given in the table 3 .

\section{Table 3. Simulation parameter values}

\begin{tabular}{|l|c|}
\hline Parameter & value \\
\hline Transmitted Power & $-30 \mathrm{dBm}$ \\
\hline sampling frequency & $50 \times 10^{9} \mathrm{~Hz}$ \\
\hline frame time & $5 \times 10^{-9} \mathrm{~s}$ \\
\hline Transmission bits & 1000 \\
\hline Pulses per bit & 5 \\
\hline Time slots per frame & 5 \\
\hline PPM shift & $0.5 \times 10^{-9} \mathrm{~s}$ \\
\hline
\end{tabular}

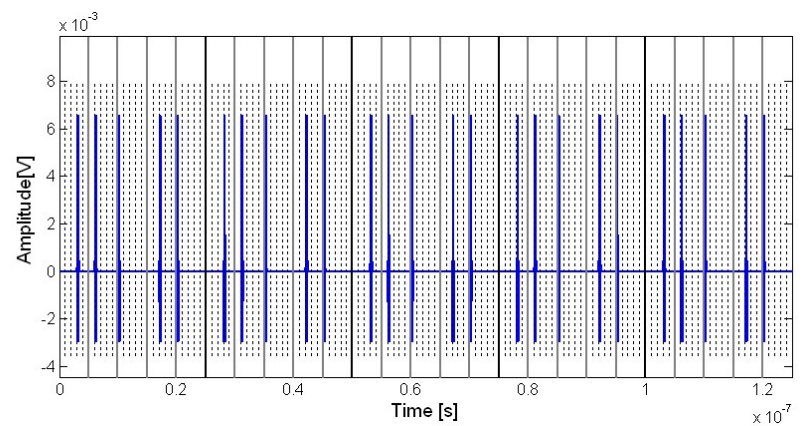

Bit 0 1 1 0

Figure 17. Reference transmitted signal using the fifth derivative Gaussian pulse

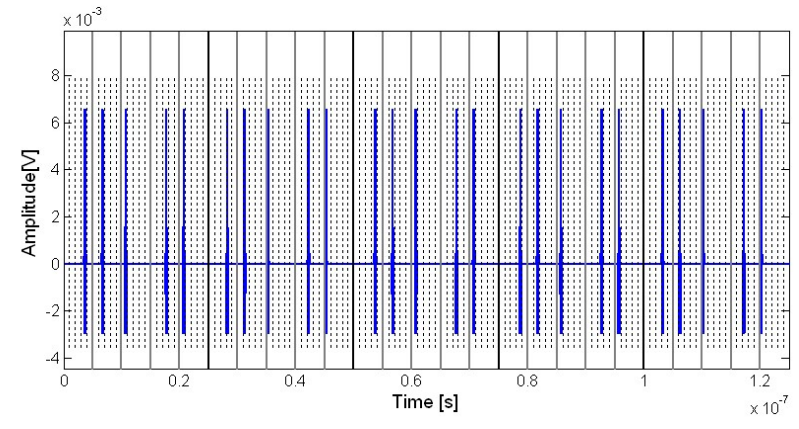

$\begin{array}{llllll}\text { Bit } & 1 & 0 & 1 & 1 & 0\end{array}$

Figure 18. Transmitted signal using the fifth derivative Gaussian pulse after Modulation

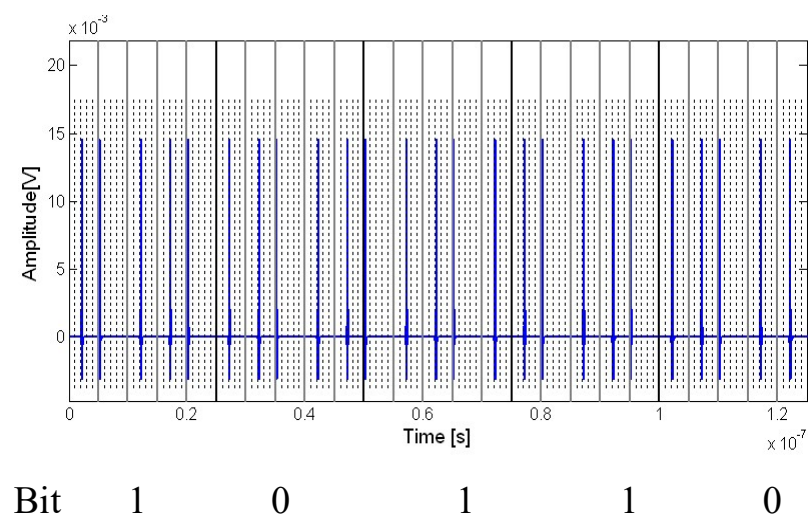

Figure 19. Reference transmitted signal using Proposed optimal combined pulse

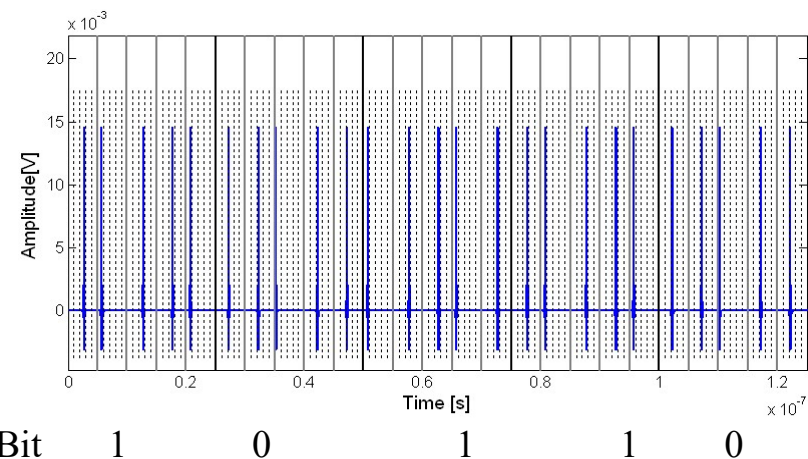

Figure 20. Transmitted signal using proposed optimal combined pulse after Modulation

Fig. 17 and Fig. 19 illustrate the transmitted reference signal before modulation and Fig. 18 and Fig. 20 show the transmitted signal after modulation using the fifth derivative Gaussian pulse and proposed method of pulse combining respectively using binary pulse position modulation time hopping technique. 
Here, five pulses are used for every transmitted bit. The time frame duration $\left(25 \times 10^{-9} \mathrm{~s}\right)$ of each bit is divided into five-time slots. Only one pulse is transmitted successfully in each time slot showing a perfect generation of time hopping signals.

The modulated signal in Fig. 18 and Fig. 20 show a pulse position shift of $0.5 \times 10^{-9} \mathrm{~s}$ for the transmitted bit 1 used to modulate the reference combined signal. The pulse shift is clearly visible for every binary bit 1 to be transmitted and no pulse shift is observed for the transmission of binary bit 0 . Although the transmission and reception are done for one thousand random transmission bits. But, for the clear illustration of the perfect generation of the transmitted signal with the pulse shift for every binary bit 1 the figures are shown for five bit random sequence. From the above figures, we can conclude that a perfect transmission signal is generated using our proposed approach of pulse combining.

The rake receiver results for Ideal rake, selective rake, and Partial rake receiver configurations using two rake receiver branches are plotted as bit error rate (BER) versus energy per bit to noise power spectral density ratio (normalized signal to noise ratio) in decibel $(\mathrm{Ex} / \mathrm{No} \mathrm{dB})$ for the standard gaussian fifth derivative pulse shape and the proposed combined pulse shape using using optimal pulse shape factors, after passing the transmitted signal through the UWB channel model CM2 and CM3 shown in Fig. 21- Fig. 24.

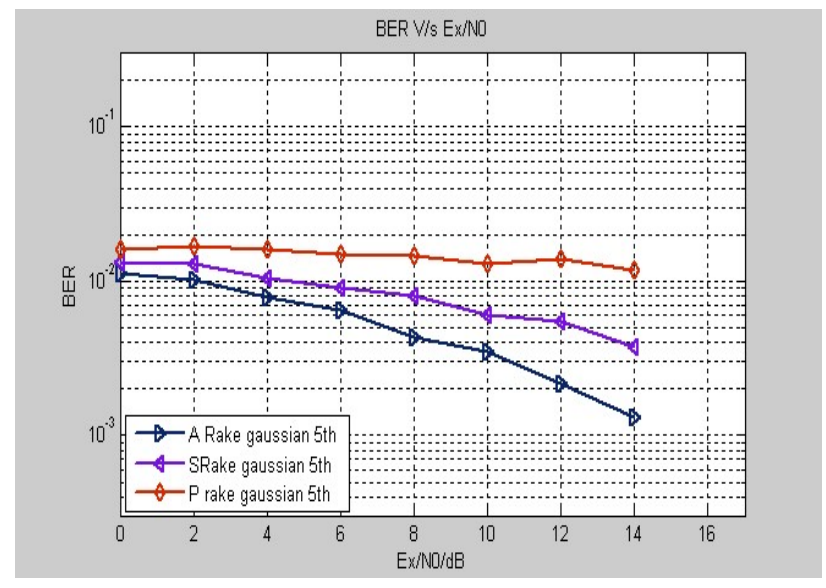

Figure 21. BER Analysis of System Model for the standard Gaussian fifth derivative using optimal coefficients in Channel Model CM2

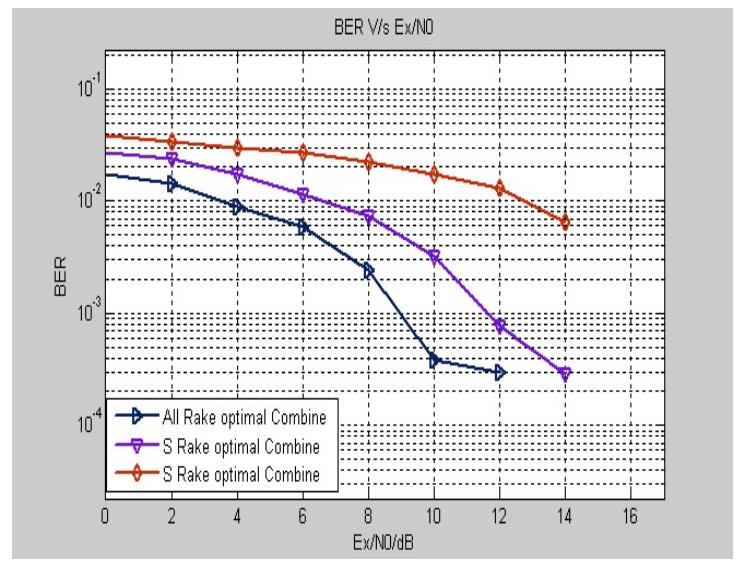

Figure 22. BER Analysis of System Model for the optimal proposed combined pulse using optimal coefficients in Channel Model CM2

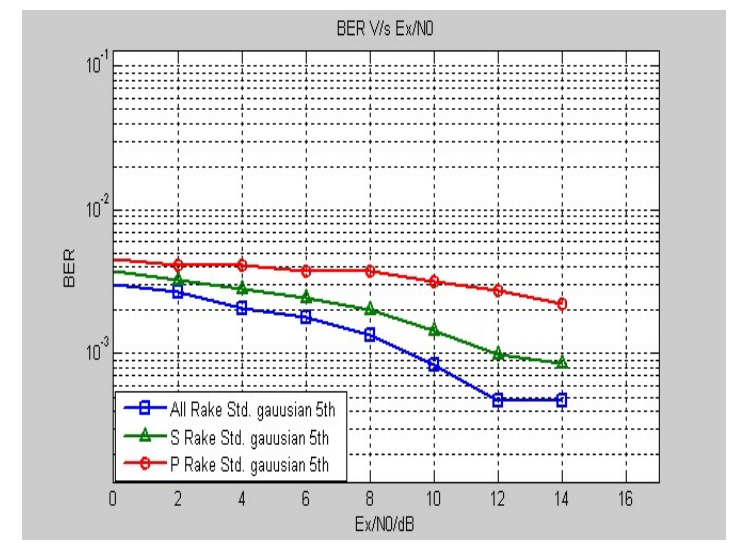

Figure 23. BER Analysis of System Model for the standard Gaussian fifth derivative using optimal coefficients in Channel Model CM3

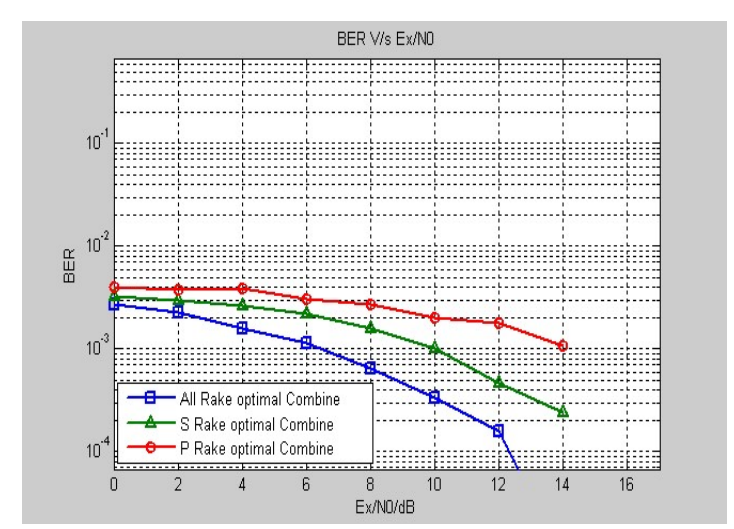

Figure 24. BER Analysis of System Model for the optimal proposed combined pulse using optimal coefficients in Channel Model CM3 


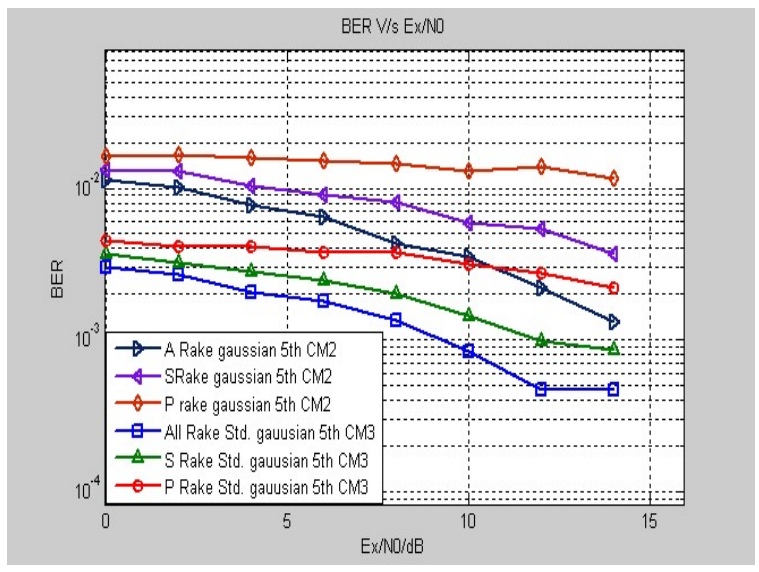

Figure 25. BER Comparison Analysis of System Model for the standard Gaussian fifth derivative using optimal coefficients in Channel Model CM2 and CM3

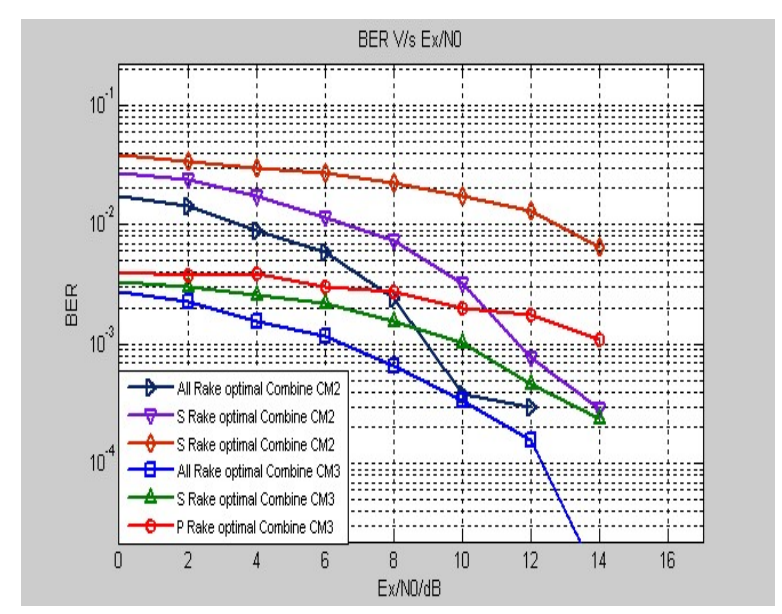

Figure 26. BER Comparison Analysis of System Model for the proposed combined pulse using optimal coefficients in Channel Model CM2 and CM3

From the Fig. 25 and Fig. 26. it is concluded that the Gaussian fifth derivative pulse and optimal proposed combined pulse with optimal pulse shape factors perform better in terms of bit rate over Non line of sight channel model 3 for the three rake receivers (A rake, Selective rake, and partial rake). So, in our research work, we will use channel model CM3 as a model to obtain improved results.

Based on the BER Simulation comparison results of the Gaussian derivative pulse and the optimal proposed combined pulse using pulse shape factors shown in Fig. 27., we can see that the Ideal (All rake) shown dark-blue curve, selective rake shown by the purple curve, and partial rake receiver shown by brown curve for the optimal combined pulse show better performance in terms of the bit error rate over the Ideal (All rake) shown with a sky pink curve, selective rake shown by the green curves, and a partial rake receiver shown by red curve for the Gaussian $5^{\text {th }}$ derivative pulse with optimal pulse factors respectively. The results obtained above in terms of bit error rates at different normalized signal to noise ratio $(\mathrm{Ex} / \mathrm{No} \mathrm{dB})$ values are given in the Table 4.

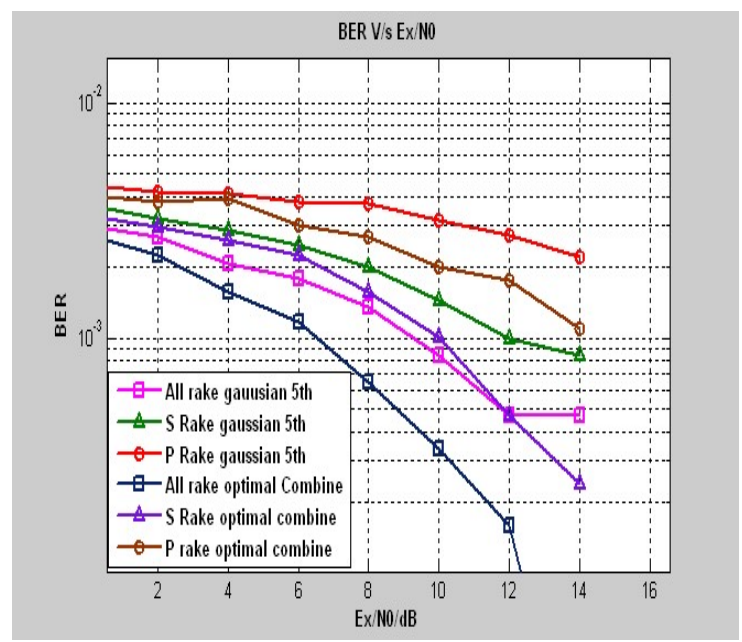

Figure 27. BER Comparison Analysis of System Model for the standard Gaussian fifth derivative and proposed combined pulse using optimal coefficients in multipath channel CM3

The bit error rate values obtained for All RAKE (ARAKE) Selective RAKE (S-RAKE) and Partial RAKE (P-RAKE) receivers respectively obtained in the table 4, using Time Hopping UWB system with Gaussian fifth derivative pulse and the proposed pulse using modified random combination of pulses with optimal pulse shape factors at $0 \mathrm{~dB}, 6 \mathrm{~dB}$ and 10 $\mathrm{dB}$ normalized signal to noise ratio $((\mathrm{Ex} / \mathrm{No}) \mathrm{dB})$ provides better results than

i. The bit error rate values of About $2.04 \times 10^{-1}$ for A- RAKE, $3.2 \times 10^{-1}$ for S- RAKE, $3.8 \times 10^{-1}$ for PRAKE at $0 \mathrm{~dB}$ and $5 \times 10^{-2}$ for A- RAKE, $1.9 \times 10^{-}$ ${ }^{1}$ for S-RAKE and $2.7 \times 10^{-1}$ for P-RAKE at $6 \mathrm{~dB}$ normalized signal to noise ratio for pulse position modulation Time Hopping UWB system with the Gaussian derivative pulse with shaping factor of $0.4 \times 10^{-9} \mathrm{~s}$, presented in [29]

ii. The bit error of about $7.9 \times \times 10^{-3}$ and $8 \times 10^{-4}$ at $6 \mathrm{~dB}$ and $10 \mathrm{~dB}$ normalized signal to noise ratio $((\mathrm{Ex} / \mathrm{No}) \mathrm{dB})$ respectively for the Gaussian derivative pulse Ultra wideband system with 
shaping value of $1 \times 10^{-9} \mathrm{~s}$ presented in [22].

iii.Bit error rate results of about $1.7 \times 10^{-1}$ for ALL

RAKE, $2.2 \times 10^{-1}$ for S-RAKE and $2.8 \times 10^{-1}$ for PRAKE at $0 \mathrm{~dB}\left(\left(\mathrm{Ex} / \mathrm{No}_{\mathrm{o}}\right) \mathrm{dB}\right)$, about $3.3 \times 10^{-2}$ for ALL RAKE, $4.5 \times 10^{-2}$ for S-RAKE and $7.9 \times 10^{-2}$ for P-RAKE at $6 \mathrm{~dB}\left(\left(\mathrm{Ex}_{\mathrm{No}}\right) \mathrm{dB}\right)$ about $4.4 \times 10^{-3}$ for ALL RAKE, $8.2 \times 10^{-3}$ for S-RAKE and 2.02 $\mathrm{x} 10^{-2}$ for P-RAKE at $10 \mathrm{~dB}$ normalized signal to noise ratio [24].

iv. The bit error rate of about $1.6 \times 10^{-1}, 3.2 \times 10^{-2}$ and
$4.2 \times 10^{-3}$ at $0 \mathrm{~dB}, 6 \mathrm{~dB}$ and $10 \mathrm{~dB}$ normalized signal to noise ratio respectively, combination of the Gaussian pulse derivatives in the UWB spectrum regions with same shaping factor of $0.7 \times 10^{-9} \mathrm{~s}$ presented in [14].

v.Bit error rate of about $7.2 \times 10^{-2}$ presented in S. K Shaikhah et al. (2015) for Ultra wideband system using the Gaussian fifth derivative pulse with shaping value of $1.25 \times 10^{-10} \mathrm{~s}$ [31].

Table 4. Bit error rates at different normalized signal to noise ratio (Ex/No dB) values

\begin{tabular}{|c|c|c|c|c|}
\hline \multirow{2}{*}{ UWB Pulse } & \multirow{2}{*}{ Receiver Configuration } & \multicolumn{3}{|c|}{ BER at different (Ex/No) dB values } \\
\hline & & $0 \mathrm{~dB}$ & $6 \mathrm{~dB}$ & $10 \mathrm{~dB}$ \\
\hline \multirow{3}{*}{$\begin{array}{l}\text { PPM Time Hopping UWB System Using } \\
\text { Gaussian fifth derivative Pulse using } \\
\text { optimal values of pulse shape factor }\end{array}$} & Ideal RAKE (ARAKE) & $3.02 \times 10^{-3}$ & $1.79 \times 10^{-3}$ & $8.4 \times 10^{-4}$ \\
\hline & $\begin{array}{c}\text { Selective RAKE (S- } \\
\text { RAKE) }\end{array}$ & $3.72 \times 10^{-3}$ & $2.46 \times 10^{-3}$ & $1.44 \times 10^{-3}$ \\
\hline & $\begin{array}{r}\text { Partial R } \\
\text { (P-RAl }\end{array}$ & $4.51 \times 10^{-3}$ & $3.77 \times 10^{-3}$ & $3.18 \times 10^{-3}$ \\
\hline \multirow{3}{*}{$\begin{array}{l}\text { PPM Time Hopping UWB System Using } \\
\text { Modified Combination of Pulses using } \\
\text { optimal values of pulse shape factors }\end{array}$} & Ideal RAKE (ARAKE) & $2.76 x$ & $1.16 \times 10^{-3}$ & $3.4 \times 10^{-4}$ \\
\hline & $\begin{array}{c}\text { Selective RAKE (S- } \\
\text { RAKE) }\end{array}$ & $3.29 \times 10^{-3}$ & $2.23 \times 10^{-3}$ & $1.01 \times 10^{-3}$ \\
\hline & $\begin{array}{l}\text { Partial RAKE } \\
\text { (P-RAKE) }\end{array}$ & $4.04 \times 10^{-3}$ & $3.04 \times 10^{-3}$ & $2.03 \times 10^{-3}$ \\
\hline
\end{tabular}

\section{Conclusion}

This paper presents the proposed random combination approach of signal generation to be used for ultra wideband communication systems which can provide efficient results in meeting the regulations for the transmitting pulse set by the regulatory authorities. The new combined pulse using the first five Gaussian derivative pulses with optimal shaping factors as basic functions have been generated successfully with the channel coefficients and pulse shaping factors meeting the power spectral density requirements of an ultra-wideband signal.

The transmission and reception ultrawideband system model are created and simulated in Matlab using binary modulation and pulse position modulation time hopping multiple access modulation scheme in the presence of IEEE UWB multipath Non line of Sight channel model CM2 and CM3. The rake receiver results plotted as bit error rate (BER) versus normalized energy per bit to noise power spectral density ratio in decibel shows that the combined transmitted pulse UWB system and the Gaussian fifth derivative pulse system using optimal pulse shape factors showed better performance over Non line of sight multipath channel model 3 for the three rake receivers. Also, the proposed optimal combined Pulse shape multiple access system gives an improved performance in terms of bit error rate at different normalized signal to noise ratio $(\mathrm{Ex} / \mathrm{No} \mathrm{dB})$ values over the Gaussian fifth derivative pulse for A rake, selective rake, and partial rake receiver configurations. The proposed pulse ultra wideband system also showed improved results as compared to the previous literature results. This optimal pulse can be used as a reference for achieving improved results in multipath channel environments.

\section{References}

[1] US Federal Communications Commission: FCC Revision of part 15 of the commission's rules regarding ultra-wideband transmission systems First report and order, Technical report, 2002, 1-89.

[2] Goyal, V.: Pulse Generation and Analysis of Ultra Wide Band System Model, Computer Science \& Telecommunications, 34 (2012), 3-6.

[3] Goyal, V., Dhaliwal, B. S: Optimal Pulse Generation for the improvement of ultra wideband system performance, Recent 
Advances in Engineering and Computational Sciences (RAECS), 2014, 1-6.

[4] Ali, T., Siddiqua P., Matin, MA.: Performance evaluation of different modulation schemes for ultra wide band systems, Journal of ELECTRICAL ENGINEERING, 65 (2014), 3, 184-188.

[5] Lin, B., Cai, G., Zhuang, M.: Capacity comparison of UWB system based on combined Cosinusoid Gaussian pulse, In AntiCounterfeiting, Security and Identification (ASID), IEEE International Conference, 2011, 93-96.

[6] Li, Bin, Zhou, Z., Zou, W., Li, D., Zhao, D.: Optimal waveforms design for ultra-wideband impulse radio, sensors, 10 (2010), 12, 1103811063.

[7] Koonen, M. J.: Novel generation and transmission of 2 Gbps impulse radio ultra wideband over MMF for in-building networks application, Optical Fiber Communication (OFC) collocated National Fiber Optic Engineers Conference, 2010, 1-3.

[8] Wang, L. Li. Wu, P. X. d., Zhang, J.: Improved UWB pulse shaping method based on Gaussian derivatives. Wireless Mobile and Computing IET International Communication Conference on, Shanghai, 2011, 438-442.

[9] Pengxiao, Li., Hongwei, C., Minghua, C., Shizhong, X.: Gigabit/s photonic generation, modulation, and transmission for a reconfigurable impulse radio UWB over fiber system. IEEE photonics journal, 4 (2012), 805816.

[10] Mirshafiei, M., Abtahi, M., Rusch, L. A.: Ultrawideband pulse shaping: bypassing the inherent limitations of the Gaussian monocycle, IET communications, 6 (2012), 1068-1074.

[11] Wang, S. B., Zheng Zhou, and Kyungsup Kwak. Two pulse designs for ultra wideband-cognitive radio by using multiple modified transform domain communication system. Applied Mathematics and Information Sciences, 2011, 6 (3), 619-628.

[12] Abraha, S. T., Okonkwo, C., Gamage, P. A., Tangdiongga, E., Koonen, T.: Routing of power efficient IR-UWB wireless and wired services for in-building network application, Journal of Lightwave Technology, 30 (2012), 1651-1663.

[13] Menon, M. B., Gopakumar, A., Iqbal, N. V.: A hybrid approach for UWB pulse shaping, 2nd International Conference on Electronics and Communication Systems IEEE, 2015, 373-377.
[14] Rinija, G. N., Menon, M. B., Iqbal, N. V., Gopakumar, A.: Interference Mitigation Techniques Using UWB Pulse Shaping, Procedia Technology, 24 (2016), 804811.

[15] Goyal, Vikas, Dhaliwal, B.S.: Ultra Wideband PAM Modulation and Reception in UWB Multi Path Channel Using Rake Configurations, Computer Science \& Telecommunications, 45 (2015), 1, 71-76.

[16] Goyal, Vikas, Dhaliwal, B.S.: Ultra Wideband Pulse Modulation System in Comparison to Conventional Narrowband Wireless Systems, 19th Punjab Science Congress, S.U.S Group of Institutes, Tangori, 2016, 179-180.

[17] Filianau, A., Zhe, W.: Channel Characterization and Modulation Schemes Of Ultra Wideband Systems, International Journal of Mobile Network Communications \& Telematics, (2014), 425-32.

[18] Molisch, A. F., Cassioli, D.: A Comprehensive Standardized Model for Ultrawideband Propagation Channels. IEEE Transactions on Antennas and Propagation, 54 (2006), 31513166.

[19] Zhang, H., Aaron, G. T.: Performance and Capacity of PAM and PPM UWB TimeHopping Multiple Access Communications with Receive Diversity, EURASIP Journal on Advances in Signal Processing, 3 (2005), 1-10.

[20] Zuo, X. Wang, D., Yao R.: RAKE reception for IR-UWB systems in high mobile environments, Proc. International Conference on Systems and Informatics, 2012, 1459-1462.

[21] Fayadh, R. A., Malek, F., Hilal, A., Fadhil, N. S.: Improved Rake Receiver Based On the Signal Sign Separation in Maximal Ratio Combining Technique for Ultra Wideband Wireless Communication, International Journal of Electrical, Robotics Electronics and Communications Engineering, 7 (2013), 12-19.

[22] Taran, S., Nitnaware, D.: Performance Evaluation of 802.15.3a UWB Channel Model with Antipodal, Orthogonal and DPSK Modulation Scheme, I.J. Wireless and Microwave Technologies, 1 (2016), 34-42.

[23] Goyal, Vikas, Dhaliwal, B. S.: Ultra Wideband Pulse Generation Using Multiple Access Modulation Schemes, International Journal of Engineering Sciences \& Research Technology, 4 (2015), 53-59.

[24] Xiao-fei, K., Bai-ping, L.: The BER performance analysis and simulation of rake 
receiver for UWB systems, Advanced Research and Technology in Industry Applications, IEEE Workshop, 2014, 267-269.

[25] Vujovic1, I., Soda1, J., Kuzmanic, I.: Doppler Spectrum Type Contribution to BER in Fiber Optic Communication Channel, Engineering Review, 36 (2016), 1, 71-79.

[26] Foerster, J. R.: The effects of multipath interference on the performance of UWB systems in an indoor wireless channel, IEEE 53rd Vehicular Technology Conference, 2005, 1176-1180.

[27] Priya, S.S., Alamelu, N.R.: Design Considerations of UWB Gaussian pulse for Channel Estimation, International Journal of Research in Engineering and Technology, 2 (2013), 65-68.

[28] Akbar, R., Emanuel, R.: An overview of synchronization algorithms for IR-UWB systems, Proc. International Conference on Computing Networking and Communications, 2012, 573-577.

[29] Cracuin F.: UWB Rake Receiver Performance Analysis for Two Different Modulation Techniques, Scientific Bulletin Series C Electrical Engineering and Computer Science, 75 (2013), 151-162.

[30] Abraha, S. T., Okonkwo, C., Yang, H., Visani, D., Shi, Y., Jung, Hyun-Do, Tangdiongga, E., Koonen, T.: Performance evaluation of IRUWB in short range fiber communication using linear combination of monocycles, Journal of Lightwave Technology, 29 (2011), 1143-1151.

[31] Shaikhah, S. K., Mustafa, S. A., Al-sulaifanie, B. K.: Performance Study of UWB System at Various Signal Parameters. Indian Journal of Applied Research, 5 (2016), 126-129. 